\title{
Hydroxychloroquine and chloroquine for COVID-19 infection. Rapid systematic review
}

\section{Rafael Leite Pacheco ${ }^{1}$ Rachel Riera ${ }^{2}$ (1)}

${ }^{1}$ Corresponding author. Universidade Federal de São Paulo (São Paulo), Centro Universitário São Camilo (São Paulo). São Paulo, Brazil. rleitepacheco@hotmail.com ${ }^{2}$ Núcleo de Avaliação de Tecnologias em Saúde, Hospital Sírio-Libanês (São Paulo), Universidade Federal de São Paulo (São Paulo). São Paulo, Brazil. rachelriera@hotmail.com

\begin{abstract}
CONTEXT: Based on the results of preliminary studies, the off-label use of hydroxychloroquine for COVID-19 infection has been observed in practice. OBJECTIVES: To identify, systematically assess and summarize the best available evidence on the efficacy and safety of the use of hydroxychloroquine and chloroquine for COVID-19 infection. METHODS: Rapid systematic review. RESULTS: After the selection process, 30 studies were included: one open-label randomized trial, one open-label non-randomized trial and 28 ongoing studies. The outcome 'detection of viral load in oral swab' (surrogate outcome) was evaluated by both studies, involving a total of 72 participants. The findings of the studies were discordant: one study observed a higher frequency of negative viral load associated with hydroxychloroquine on day-7, while the other study did not observe any difference between hydroxychloroquine and the control group (standard treatment) on day- 6 . Both studies have methodological limitations when evaluated by specific tools according to study design (Cochrane Bias Risk Table and ROBINS-I). CONCLUSION: This rapid systematic review identified two clinical studies (with available data), with limited methodological quality, that evaluated the effects of hydroxychloroquine for COVID-19 infection. Based on the findings of these two studies, the efficacy and safety of hydroxychloroquine and chloroquine in patients with COVID-19 is still uncertain (very low evidence certainty) and its routine use for this situation should not be recommended until the results of ongoing studies could provide a proper assessment of their effects.
\end{abstract}

KEYWORDS: COVID-19. SARS-CoV-2. Coronavirus. Hydroxychloroquine. Chloroquine. Systematic review 


\section{Introduction}

Since December 2019, when the first outbreak of COVID-19 infection was revealed in China (Wuhan, Hubei province), researches have been conducted to discover rapid and accurate diagnosis tests, to develop vaccines and to assess therapeutic options for the treatment and prevention of this disease and its complications, as SARS-Cov-2.

Funding agencies are prioritizing resources for several studies that aim to elucidate the epidemiological features, pathophysiology, risk factors, prognosis and clinical evolution of the emerging virus.

The COVID-19 pandemics has mobilized research organizations, database, renowned publishers and editorial groups - as examples the Royal Society of Tropical Medicine and Hygiene, Elsevier, Cochrane, University of Oxford and British Medical Journal which have been currently working to provide open access scientific content of COVID-19 for healthcare professionals and general population.

In front of a pandemic, all those actions are expected and must be recognized as legitimates attempts to minimize the consequences of a new disease that seems to be highly transmissible and associated with major complications, elevated number of admissions on intensive care units, high costs and resources consumption, and an unpredictable economic impact worldwide.

However, the expectation that 'new discoveries' can substantially change this uncertain scenario should be based on reliable and objective data. The expectation should not ignore or underestimate the methodological rigor of the available researchers, and it's necessary to differentiate the obvious from the evidence and the pathophysiological rationale from the results of a well-planned and -conducted clinical trial.

Based on preliminary data, healthcare authorities have recommended the use of hydroxychloroquine or chloroquine for treating COVID-191-3.

The scarcity of these drugs for those patients with diseases for which they are formally indicated including chronic autoimmune diseases such as lupus erythematosus and rheumatoid arthritis - is already a reality.
In order to scientifically and impartially inform health decision making, a rapid systematic review was developed to map and critically assess the best existing evidence on the use of hydroxychloroquine and chloroquine for COVID-19 infection.

\section{Objectives}

To identify, systematically appraise and summarize the available scientific evidence on the efficacy and safety of the use of hydroxychloroquine and chloroquine for COVID-19 infection.

Structured research question (PICO acronym):

- $\quad \mathrm{P}$ (population): people with suspect or confirmed COVID-19 infection.

- I (intervention): hydroxychloroquine or chloroquine (isolated or combined with other interventions).

- C (comparators): general health support care, placebo, no specific intervention or any other active treatment.

- O (outcomes): efficacy and safety outcomes detailed under the methods section.

- S (studies): clinical studies or secondary studies that considered clinical studies as an inclusion criteria.

\section{Methods}

\section{Study design and setting}

This was a rapid systematic review developed at Center of Health Technology Assessment, Hospital Sírio-Libanês in collaboration with the Discipline of Economics and Health Management, Universidade Federal de São Paulo (Unifesp), São Paulo - Brazil. This review was conducted in accordance with the recommendations of the Cochrane Handbook for Systematic Reviews of Interventions ${ }^{4}$. This manuscript was written following the PRISMA Statement ${ }^{5}$. Since this was a rapid systematic review, a register on the PROSPERO database has not been consolidated. 


\section{Eligibility criteria}

(a) Types of participant types

Adults and children with suspected or confirmed diagnosis of COVID-19 infection.

(b) Types of interventions

Hydroxychloroquine or chloroquine alone or in combination with other interventions.

(c) Types of studies

Taking into account the limited number of studies that may have been published so far and that the purpose of this review is to map the current knowledge, the following study designs were considered, following the hierarchy of evidence and considering their methodological quality: randomized clinical trials, quasi-randomized clinical trials, non-randomized clinical trials, cohort studies, case-control studies, single-arm experimental cohort studies (phase 1 or 2).

\section{Outcomes of interest}

We consider any clinical and laboratory outcomes as reported by the included studies, prioritizing the following:

\section{Primary outcomes}

- Mortality related to COVID-19.

- Severe adverse events.

- Progression to COVID-19 acute respiratory syndrome (SARS-Cov-2).

\section{Secondary outcomes}

- All-cause mortality

- Admission to an intensive care unit

- Any adverse event

- Health-related quality of life

\section{Tertiary outcomes}

- Laboratory outcomes

\section{Searching for studies}

\section{Electronic search}

An electronic search was performed in the following general databases:

- Cochrane Library (via Wiley);

- Embase (via Elsevier);

- Literatura Latino-Americana e do Caribe em Ciências da Saúde (LILACS, via Biblioteca Virtual em Saúde, BVS)

- Medical Literature Analysis and Retrieval System Online (MEDLINE, via PubMed).

An electronic search was performed on the following grey literature database:

- Opengrey ( https://opengrey.eu)

An electronic search was performed in the following clinical trial registry databases:

- ClinicalTrials.gov (https://clinicaltrials.gov)

- International Clinical Trials Register Platform (ICTRP), World Health Organization (WHO), which includes among other the Chinese Clinical Trial Registry (https://www.chictr.org.cn).

The search strategies developed and ran for each electronic database are presented in Chart 1. No restrictions on date, language or status (abstract or full text) of the publication were imposed. The searches were carried out on March 19th and updated on March 26th, 2020 (with the exception of ICTRP which was temporarily inactive). 
Chart 1. Search strategies for electronic databases and other sources (to be continued)

\begin{tabular}{|c|c|c|}
\hline Database & Search strategy & Results \\
\hline Cochrane Library & $\begin{array}{l}\text { \#1 MeSH descriptor: [Coronavirus] explode all trees } \\
\text { \#2 "COVID-19" OR (COVID) OR (Coronavirus) OR (SARS-CoV-2) OR } \\
\text { (Coronaviruses) OR (Deltacoronavirus) OR (Deltacoronaviruses) OR "Munia } \\
\text { coronavirus HKU13" OR (Coronavirus HKU15) OR (Coronavirus, Rabbit) OR } \\
\text { (Rabbit Coronavirus) OR (Coronaviruses, Rabbit) OR (Rabbit Coronaviruses) OR } \\
\text { "Bulbul coronavirus HKU11" OR "Thrush coronavirus HKU12" } \\
\text { \#3 \#1 OR \#2 } \\
\text { \#4 MeSH descriptor: [Hydroxychloroquine] explode all trees } \\
\text { \#5 MeSH descriptor: [Hydroxychloroquine] explode all trees } \\
\text { \#6 MeSH descriptor: [Antimalarials] explode all trees } \\
\text { \#7 (Hydroxychloroquine) OR (Oxychlorochin) OR (Oxychloroquine) OR } \\
\text { (Hydroxychlorochin) OR (Plaquenil) OR (Hydroxychloroquine Sulfate) OR } \\
\text { "Hydroxychloroquine Sulfate (1:1) Salt" OR (Hidroxicloroquina) OR } \\
\text { (Hydroxychloroquine) OR (Hydroxychloroquinum) OR (Oxichlorochine) OR } \\
\text { (Oxichloroquine) OR Chlorochin OR Cloroquina OR Cloroquine OR Chloroquine } \\
\text { OR (Antimalarials) OR (Antimalarial Agents) OR (Agents, Antimalarial) OR } \\
\text { (Antimalarial Drugs) OR (Drugs, Antimalarial) OR (Anti-Malarials) OR (Anti } \\
\text { Malarials) OR "(N4-(7-Chloro-4-quinolinyl)-N1,N1-diethyl-1,4-pentanediamine)" } \\
\text { OR Hydroquin OR Axemal OR Dolquine OR Quensyl OR Quinoric OR Plaquenil } \\
\text { \#8 \#4 OR \#5 OR \#6 OR \#7 } \\
\text { \#9 \#3 AND \#8 }\end{array}$ & 2 \\
\hline Embase & $\begin{array}{l}\text { \#1 'coronavirinae' OR 'coronavirinae'/exp OR coronavirinae OR 'corona } \\
\text { virus'/exp OR 'corona virus' OR 'coronavirus'/exp OR coronavirus OR 'covid-19' } \\
\text { OR covid OR 'sars-cov-2' OR coronaviruses OR deltacoronavirus OR } \\
\text { deltacoronaviruses OR 'munia coronavirus hku13' OR 'coronavirus hku15' OR } \\
\text { 'coronavirus, rabbit' OR 'rabbit coronavirus' OR 'coronaviruses, rabbit' OR 'rabbit } \\
\text { coronaviruses' OR 'bulbul coronavirus hku11' OR 'thrush coronavirus hku12' }\end{array}$ & 24 \\
\hline
\end{tabular}


\#2 'hydroxychloroquine' OR 'hydroxychloroquine'/exp OR hydroxychloroquine OR ' 7 chloro 4 [4 [ethyl (2 hydroxyethyl) amino] 1 methylbutylamino] quinoline'/exp OR '7 chloro 4 [4 [ethyl (2 hydroxyethyl) amino] 1 methylbutylamino] quinoline' OR '7 chloro 4 [4 [ethyl (2 hydroxyethyl) amino] 1 methylbutylamino] quinoline diphosphate'/exp OR '7 chloro 4 [4 [ethyl (2 hydroxyethyl) amino] 1 methylbutylamino] quinoline diphosphate' OR 'chloroquinol'/exp OR chloroquinol OR 'ercoquin'/exp OR ercoquin OR 'hydrochloroquine'/exp OR hydrochloroquine OR 'hydrocloroquine'/exp OR hydrocloroquine OR 'oxychloroquine'/exp OR oxychloroquine OR 'quensyl'/exp OR quensyl OR 'sn 8137'/exp OR 'sn 8137' OR oxychlorochin OR hydroxychlorochin OR plaquenil OR 'hydroxychloroquine sulfate' OR 'hydroxychloroquine sulfate (1:1) salt' OR hidroxicloroquina OR hydroxychloroquinum OR oxichlorochine OR oxichloroquine OR 'chloroquine' OR 'chloroquine'/exp OR chloroquine OR '4 (4 diethylamino 1 methylbutylamino) 7 chlorchinolin diphosphate' OR ' 4 (4 diethylamino 1 methylbutylamino) 7 chlorchinolin sulfate' OR '4 (4 diethylamino 1 methylbutylamino) 7 chlorchinolin sulfate' OR '4 (4 diethylamino 1 methylbutylamino) 7 chloroquinoline' OR ' 7 chloro 4 (4 diethylamino 1 methylbutylamino) quinoline' OR '7 chloro 4 (4 diethylamino 1 methylbutylamino) quinoline diphosphate' OR '7 chloro 4 (4 diethylamino 1 methylbutylamino) quinoline' OR 'a-cq' OR amokin OR amokine OR anoclor OR aralan OR aralen OR 'aralen hydrochloride' OR 'aralen phosphate' $O R$ aralene $O R$ arechin $O R$ arechine $O R$ arequine $O R$ arthrochin $O R$ arthrochine $O R$ arthroquine $O R$ artrichin $O R$ artrichine $O R$ artriquine $O R$ avloclor OR avoclor OR bemaphata OR bemaphate OR bemasulph OR bipiquin OR cadiquin OR chemochin OR chemochine OR chingamine OR chingaminum OR chloraquine OR chlorochin OR chlorochine OR chlorofoz OR chloroquin OR 'chloroquin phosphate' OR 'chloroquine diphosphate' OR 'chloroquine disulfate' OR 'chloroquine disulphate' OR 'chloroquine hydrochloride' OR 'chloroquine phosphate' OR 'chloroquine streuli' OR 'chloroquine sulfate' OR 'chloroquine sulphate' OR chloroquinesulphate OR 'chloroquini diphosphas' OR 'chloroquinum diphosphoricum' OR chlorquin OR chlorquine OR choloquine OR 'choroquine sulfate' OR 'choroquine sulphate' OR cidanchin OR 'clo-kit junior' OR clorichina OR clorichine OR cloriquine OR clorochina OR delagil OR delagyl OR dichinalex OR diclokin OR diquinalex OR diroquine OR emquin OR genocin OR gontochin OR gontochine OR gontoquine OR heliopar OR imagon OR iroquine OR klorokin OR klorokine OR klorokinfosfat OR lagaquin OR malaquin OR malarex OR malarivon OR malaviron OR maliaquine OR maquine OR mesylith $O R$ mexaquin $O R$ mirquin $O R$ nivachine $O R$ nivaquin $O R$ nivaquine $O R$ 'nivaquine (b)' OR 'nivaquine b' OR 'nivaquine dp' OR 'nivaquine forte' OR ' $p$ roquine' OR quinachlor $O R$ quingamine $O R$ repal $O R$ resochen $O R$ resochene $O R$ resochin $O R$ 'resochin junior' OR resochina OR resochine OR resochinon OR resoquina OR resoquine OR reumachlor OR roquine OR 'rp 3377' OR rp3377 OR sanoquin OR sanoquine OR silbesan OR siragan OR sirajan OR 'sn 7618' OR sn7618 OR solprina OR solprine OR tresochin OR tresochine OR tresoquine OR trochin OR trochine OR troquine OR ' $w$ 7618' OR w7618 OR 'win 244' OR win244 OR 'antimalarial agent'/exp OR 'antimalarial agent' OR 'anti malaria drug'/exp OR 'anti malaria drug' OR 'antimalaria agent'/exp OR 'antimalaria agent' OR 'antimalaria drug'/exp OR 'antimalaria drug' OR 'antimalaria drug, synthetic'/exp OR 'antimalaria drug, synthetic' OR 'antimalarial'/exp OR antimalarial OR 'antimalarial drug'/exp OR 'antimalarial drug' OR 'antimalarials'/exp OR antimalarials OR 'antipaludean agent'/exp OR 'antipaludean agent' OR 'antiplasmodic agent'/exp OR 'antiplasmodic agent' OR 'synthetic antimalaria agent'/exp OR 'synthetic antimalaria agent' \#3 \#1 AND \#2 \#4 \#3 AND [embase]/lim NOT ([embase]/lim AND [medline]/lim) 


\begin{tabular}{|c|c|c|}
\hline & $\begin{array}{l}\text { \#2 MH:"Hydroxychloroquine" OR MH:"Hidroxicloroquina" OR } \\
\text { MH:D03.633.100.810.050.180.350\$ OR (Hydroxychloroquine) OR } \\
\text { (Hidroxicloroquina) OR (Hydroxychlorochin) OR (Hydroxychloroquine Sulfate) } \\
\text { OR "Hydroxychloroquine Sulfate (1:1) Salt" OR (Oxychlorochin) OR } \\
\text { (Oxychloroquine) OR (Plaquenil) OR (Oxicloroquina) OR MH:"Cloroquina" OR } \\
\text { MH:"Chloroquine" OR MH:D03.633.100.810.050.180\$ OR (Cloroquina) OR } \\
\text { (Chloroquine) OR (Aralen) OR (Arechine) OR (Arequin) OR (Chingamin) OR } \\
\text { (Chlorochin) OR (Chloroquine Sulfate) OR (Chloroquine Sulphate) OR } \\
\text { (Khingamin) OR (Nivaquine) OR (Sulfate, Chloroquine) OR (Sulphate, } \\
\text { Chloroquine) OR MH:"Antimaláricos" OR MH:"Antimalarials" OR } \\
\text { MH:D27.505.954.122.250.100.085\$ OR (Antimaláricos) OR (Antimalarials) OR } \\
\text { (Agents, Antimalarial) OR (Anti Malarials) OR (Anti-Malarials) OR (Antimalarial } \\
\text { Agents) OR (Antimalarial Drugs) OR (Drugs, Antimalarial) } \\
\text { \#3 \#1 AND \#2 }\end{array}$ & \\
\hline MEDLINE & $\begin{array}{l}\text { \#1 "Coronavirus"[Mesh] OR "COVID-19" OR (COVID) OR (Coronavirus) OR (SARS- } \\
\text { CoV-2) OR (Coronaviruses) OR (Deltacoronavirus) OR (Deltacoronaviruses) OR } \\
\text { "Munia coronavirus HKU13" OR (Coronavirus HKU15) OR (Coronavirus, Rabbit) } \\
\text { OR (Rabbit Coronavirus) OR (Coronaviruses, Rabbit) OR (Rabbit Coronaviruses) } \\
\text { OR "Bulbul coronavirus HKU11" OR "Thrush coronavirus HKU12" } \\
\text { \#2 "Hydroxychloroquine"[Mesh] OR (Hydroxychloroquine) OR (Oxychlorochin) } \\
\text { OR (Oxychloroquine) OR (Hydroxychlorochin) OR (Plaquenil) OR } \\
\text { (Hydroxychloroquine Sulfate) OR "Hydroxychloroquine Sulfate (1:1) Salt" OR } \\
\text { (Hidroxicloroquina) OR (Hydroxychloroquine) OR (Hydroxychloroquinum) OR } \\
\text { (Oxichlorochine) OR (Oxichloroquine) OR "Chloroquine"[Mesh] OR Chlorochin } \\
\text { OR Cloroquina OR Cloroquine OR Chloroquine OR "Antimalarials"[Mesh] OR } \\
\text { (Antimalarials) OR (Antimalarial Agents) OR (Agents, Antimalarial) OR } \\
\text { (Antimalarial Drugs) OR (Drugs, Antimalarial) OR (Anti-Malarials) OR (Anti } \\
\text { Malarials) OR “(N4-(7-Chloro-4-quinolinyl)-N1,N1-diethyl-1,4-pentanediamine)" } \\
\text { OR Hydroquin OR Axemal OR Dolquine OR Quensyl OR Quinoric } \\
\text { \#3 \#1 AND \#2 }\end{array}$ & 54 \\
\hline Opengrey & $\begin{array}{l}\text { \#1 "COVID-19" OR (COVID) OR (Coronavirus) OR (SARS-CoV-2) OR } \\
\text { (Coronaviruses) OR (Deltacoronavirus) OR (Deltacoronaviruses) }\end{array}$ & 76 \\
\hline ClinicalTrials.gov & $\begin{array}{l}\text { \#1 "COVID-19" OR (COVID) OR (Coronavirus) OR (SARS-CoV-2) OR } \\
\text { (Coronaviruses) OR (Deltacoronavirus) OR (Deltacoronaviruses) } \\
\text { \#2 Hydroxychloroquine OR Oxychlorochin OR Oxychloroquine OR } \\
\text { Hydroxychlorochin OR Plaquenil OR Chlorochin OR Cloroquina OR Cloroquine } \\
\text { OR chloroquine OR Antimalarials OR Antimalarial } \\
\text { \#3 \#1 AND \#2 }\end{array}$ & 12 \\
\hline WHO-ICTRP & $\begin{array}{l}\text { \#1 "COVID-19" OR (COVID) OR (Coronavirus) OR (SARS-CoV-2) OR } \\
\text { (Coronaviruses) OR (Deltacoronavirus) OR (Deltacoronaviruses) } \\
\text { \#2 Hydroxychloroquine OR Oxychlorochin OR Oxychloroquine OR } \\
\text { Hydroxychlorochin OR Plaquenil OR Chlorochin OR Cloroquina OR Cloroquine } \\
\text { OR chloroquine OR Antimalarials OR Antimalarial } \\
\text { \#3 \#1 AND \#2 }\end{array}$ & 33 \\
\hline
\end{tabular}

LILACS: Literatura Latino-Americana e do Caribe em Ciências da Saúde; MEDLINE: Medical Literature Analysis and Retrieval System Online; WHO-ICTRP: World Health Organization - International Clinical Trials Register Platform. 
A manual search was performed on the reference lists of the relevant studies.

\section{Study selection and data extraction}

The process to select the studies was performed by two independent review authors and disagreements were solved by consensus. Study selection was conducted in two steps. The first step consisted in the screening of titles and abstracts of all retrieved references. The potentially eligible references were read in full (second stage), to confirm their eligibility. The entire process was performed using Rayyan platform (https://rayyan.qcri.org) ${ }^{6}$. The procedures for data extraction were conducted by two independent review authors as well.

\section{Methodological quality/risk of bias assessment}

The methodological quality/risk of bias assessment of the included studies was performed using appropriate tools for each study design, as following:

- Randomized controlled trial: Cochrane Risk of Bias Table 4 .

- Non-randomized controlled trial or quasirandomized: ROBINS-I ${ }^{\text {? }}$.

- Longitudinal comparative observational studies (case-control and cohort): Newcastle-Ottawa ${ }^{8}$.

- For phase 1/2 clinical trials without a direct comparator arm, it would be used an adapted version of the Cochrane Risk of Bias Table ${ }^{4}$, as they are not validated tools for this study design.

\section{Unity of analysis}

The unit of analysis was the individual.

\section{Measures of treatment effect and analysis proce- dures}

According to data availability and homogeneity of studies, we would pool results by including studies through random-effects meta-analysis (quantitative synthesis). Risk ratios and mean differences would be calculated to assess dichotomous and continuous variables, respectively. A 95\% confidence interval would be considered in the analysis. The software used to perform all analysis would be Review
Manager 5.3 software. However, in this review, meta-analyses were not possible (data availability or heterogeneity of studies - reasons detailed under results section), then results were presented narratively (qualitative synthesis) considering the effect size estimates (relative risk, absolute risk difference, hazard ratio, odds ratio, number needed to treat and others) and their respective confidence or variance measures (dispersion measures, confidence intervals and $p$ values).

\section{Investigating heterogeneity}

Methodological and clinical diversity of included studies were considered when deciding conduct or not quantitative synthesis. The statistical heterogeneity would be considered by means of a Chi ${ }^{2}$ test $(p<0.1$ was used as a significance cut-off) and $\mathrm{l}^{2}$ test ( $12>50 \%$ would be used as an indicative of high inconsistency among studies). Subgroup analyses were planned to explore reasons for heterogeneity and its impacts would be discussed.

\section{Additional analyses}

We planned to perform the following additional analyses, but it was not possible due to the scarcity of data for quantitative synthesis.

\section{Sensitivity analyses}

a) Fixed-effect versus random effects model metaanalysis. When the results of fixed effect meta-analysis led to a different result, both would be reported.

b) Excluding from analysis studies at high risk of bias

c) Excluding from analysis studies with industry sponsorship.

\section{Subgroup analysis}

a) Severity of COVID-19 infection

b) Age of participants

c) Co-morbidity of participants (diabetes, cardiac conditions, immunosuppression, HIV)

\section{Publication bias assessment}

Investigation of publication bias assessment was planned to be performed by visual inspection of funnel plots, if more than 10 studies were included in a single meta-analysis. 
Authors from primary study were not contacted for missing data, taking into account the context underpinning a rapid systematic review. When necessary, missing standard deviations would be calculated using reported confidence intervals and/ or standard mean errors.

\section{Assessing the certainty of evidence}

To assess the certainty of evidence, we used the GRADE approach? for the clinical relevant outcomes and a summary of findings table would be presented using the GRADEpro GDT platform.

\section{Search Results}

The search strategies retrieved 223 references. After reading the title and abstract (first step), seven duplicate references (identical references) and 186 references in disagreement with PICOS were eliminated. Reading the full text of the 30 selected references confirmed the eligibility (second stage). The flowchart of the selection process is shown in Figure 1. After the selection process, 30 studies were included:

- a randomized clinical trial published in Chinese (the translation into English was used to carry out the analyzes of this review) ${ }^{10}$.

- a non-randomized ongoing clinical trial, with partial results ${ }^{11}$.

- 28 ongoing clinical studies (Chart 2).

Chart 2. Characteristics and methodological aspects of the ongoing studies (to be continued)

\begin{tabular}{|c|c|c|c|c|c|c|c|c|}
\hline Study & Status & $\begin{array}{c}\text { Estimate } \\
\text { start/end date }\end{array}$ & Design & Participants (n) & Intervention & Comparators & $\begin{array}{l}\text { Main interest } \\
\text { outcomes }\end{array}$ & Funding \\
\hline NCT04307693 & Recruiting & $\begin{array}{l}11 \text { March 2020/ } \\
\text { May } 2020\end{array}$ & RCT & $\begin{array}{l}\text { Participants with } \\
\text { confirmed } \\
\text { COVID-19 } \\
\text { diagnosis } \\
(150)\end{array}$ & Hydroxychloroquine & $\begin{array}{l}\text { Lopinavir/ritonavir } \\
\text { No intervention }\end{array}$ & $\begin{array}{l}\text { Laboratory } \\
\text { detection/viral load } \\
\text { Time to clinical } \\
\text { improvement } \\
\text { Time to death } \\
\text { Intensive unit care } \\
\text { or mechanical } \\
\text { ventilation } \\
\text { Progression to } \\
\text { oxygen } \\
\text { supplementation }\end{array}$ & $\begin{array}{l}\text { Asan Medical } \\
\text { Center }\end{array}$ \\
\hline NCT04318444 & $\begin{array}{l}\text { Not yet } \\
\text { recruiting }\end{array}$ & $\begin{array}{l}\text { March } \\
2020 / \text { March } \\
2020\end{array}$ & RCT & $\begin{array}{l}\text { Participants that } \\
\text { had household } \\
\text { or in-hospital } \\
\text { contact with } \\
\text { COVID-19 } \\
\text { patients } \\
(1600)\end{array}$ & Hydroxychloroquine & Placebo & $\begin{array}{l}\text { Confirmed COVID- } \\
19 \text { cases } \\
\text { Cases with } \\
\text { symptoms of } \\
\text { COVID-19 }\end{array}$ & $\begin{array}{l}\text { Columbia } \\
\text { University }\end{array}$ \\
\hline NCT04303507 & $\begin{array}{l}\text { Not yet } \\
\text { recruiting }\end{array}$ & $\begin{array}{l}\text { April 2020/April } \\
2021\end{array}$ & RCT & $\begin{array}{l}\text { Participants } \\
\text { without previous } \\
\text { cCOVID-19 } \\
\text { diagnosis } \\
(40000)\end{array}$ & $\begin{array}{l}\text { Hydroxychloroquine } \\
\text { /Chloroquine }\end{array}$ & Placebo & $\begin{array}{l}\text { Duration of } \\
\text { COVID-19 infection } \\
\text { Number of } \\
\text { asymptomatic } \\
\text { cases } \\
\text { Number of } \\
\text { symptomatic cases } \\
\text { Symptom severity }\end{array}$ & $\begin{array}{l}\text { University of } \\
\text { Oxford }\end{array}$ \\
\hline NCT04315896 & $\begin{array}{l}\text { Not yet } \\
\text { recruiting }\end{array}$ & $\begin{array}{l}23 \text { March } \\
2020 / 22 \text { March } \\
2021\end{array}$ & $\mathrm{RCT}$ & $\begin{array}{l}\text { Participants with } \\
\text { confirmed } \\
\text { COVID-19 } \\
\text { diagnosis and } \\
\text { serious } \\
\text { respiratory } \\
\text { impairment } \\
(500)\end{array}$ & Hydroxychloroquine & Placebo & $\begin{array}{l}\text { All-cause mortality } \\
\text { Length of } \\
\text { hospitalization } \\
\text { Mechanical } \\
\text { ventilation }\end{array}$ & Sanofi \\
\hline
\end{tabular}


Chart 2. Characteristics and methodological aspects of the ongoing studies (continuation)

\begin{tabular}{|c|c|c|c|c|c|c|c|c|}
\hline Study & Status & $\begin{array}{c}\text { Estimate } \\
\text { start/end date }\end{array}$ & Design & Participants (n) & Intervention & Comparators & $\begin{array}{c}\text { Main interest } \\
\text { outcomes }\end{array}$ & Funding \\
\hline NCT04323631 & $\begin{array}{l}\text { Not yet } \\
\text { recruiting }\end{array}$ & $\begin{array}{l}\text { March } \\
2020 / \text { December } \\
2020\end{array}$ & $\mathrm{RCT}$ & $\begin{array}{l}\text { Participants with } \\
\text { confirmed } \\
\text { COVID-19 } \\
\text { diagnosis } \\
(1116)\end{array}$ & Hydroxychloroquine & No intervention & $\begin{array}{l}\text { Number of } \\
\text { participants with } \\
\text { severe infection or } \\
\text { death }\end{array}$ & $\begin{array}{l}\text { Rambam Health } \\
\text { Care Campus }\end{array}$ \\
\hline NCT04318015 & $\begin{array}{l}\text { Not yet } \\
\text { recruiting }\end{array}$ & $\begin{array}{l}01 \text { April 2020/ } \\
31 \text { March } 2021\end{array}$ & $\mathrm{RCT}$ & $\begin{array}{l}\text { Health } \\
\text { professionals } \\
\text { that had contact } \\
\text { with COVID-19 } \\
\text { patients } \\
(400)\end{array}$ & Hydroxychloroquine & Placebo & $\begin{array}{l}\text { Number of } \\
\text { symptomatic cases } \\
\text { Absenteeism } \\
\text { Complications }\end{array}$ & $\begin{array}{l}\text { National Institute } \\
\text { of Respiratory } \\
\text { Diseases, Mexico / } \\
\text { Sanofi }\end{array}$ \\
\hline NCT04316377 & $\begin{array}{l}\text { Not yet } \\
\text { recruiting }\end{array}$ & $\begin{array}{l}23 \text { March } \\
2020 / 25 \text { March } \\
2023\end{array}$ & $\mathrm{RCT}$ & $\begin{array}{l}\text { Participants with } \\
\text { confirmed } \\
\text { COVID-19 } \\
\text { diagnosis, } \\
\text { hospitalized and } \\
\text { with serious } \\
\text { respiratory } \\
\text { impairment } \\
\text { (202) }\end{array}$ & Hydroxychloroquine & Usual treatment & $\begin{array}{l}\text { Mortality } \\
\text { Length of } \\
\text { hospitalization } \\
\text { Mechanical } \\
\text { ventilation } \\
\text { Length of ICU stay } \\
\text { Laboratory } \\
\text { detection/viral load }\end{array}$ & $\begin{array}{l}\text { University } \\
\text { Hospital, Akershus }\end{array}$ \\
\hline NCT04319900 & Recruiting & $\begin{array}{l}5 \text { March } 2020 / \\
25 \text { June } 2020\end{array}$ & $\mathrm{RCT}$ & $\begin{array}{l}\text { Participants with } \\
\text { confirmed } \\
\text { COVID-19 } \\
\text { diagnosis } \\
(100)\end{array}$ & $\begin{array}{l}\text { Chloroquine + } \\
\text { Favipiravir }\end{array}$ & Favipiravir & $\begin{array}{l}\text { Time to symptom } \\
\text { improvement }\end{array}$ & $\begin{array}{l}\text { Beijing Chao Yang } \\
\text { Hospital }\end{array}$ \\
\hline NCT04321616 & $\begin{array}{l}\text { Not yet } \\
\text { recruiting }\end{array}$ & $\begin{array}{l}26 \text { March } \\
2020 / \text { November } \\
2020\end{array}$ & $\mathrm{RCT}$ & $\begin{array}{l}\text { Hospitalized } \\
\text { participants with } \\
\text { confirmed } \\
\text { COVID-19 } \\
\text { diagnosis } \\
(700)\end{array}$ & Hydroxychloroquine & $\begin{array}{l}\text { Remdesivir } \\
\text { No intervention }\end{array}$ & $\begin{array}{l}\text { All-cause mortality } \\
\text { Intensive unit care } \\
\text { Mechanical } \\
\text { ventilation }\end{array}$ & $\begin{array}{l}\text { Oslo University } \\
\text { Hospital }\end{array}$ \\
\hline NCT04308668 & Recruiting & $\begin{array}{l}17 \text { March/May } \\
2020\end{array}$ & $\mathrm{RCT}$ & $\begin{array}{l}\text { Participants that } \\
\text { were exposed to } \\
\text { a COVID-19 } \\
\text { patient } \\
(3000)\end{array}$ & Hydroxychloroquine & Placebo & $\begin{array}{l}\text { Confirmed COVID- } \\
19 \text { cases } \\
\text { Severity of COVID- } \\
19 \text { infection } \\
\text { Hospitalization } \\
\text { Mortality }\end{array}$ & $\begin{array}{l}\text { University of } \\
\text { Minnesota }\end{array}$ \\
\hline NCT04321993 & $\begin{array}{l}\text { Not yet } \\
\text { recruiting }\end{array}$ & $\begin{array}{l}\text { March 2020/ } \\
\text { June } 2021\end{array}$ & $\mathrm{nRCT}$ & $\begin{array}{l}\text { Hospitalized } \\
\text { participants with } \\
\text { confirmed } \\
\text { COVID-19 } \\
\text { diagnosis with } \\
\text { moderate/severe } \\
\text { diasease } \\
(1000)\end{array}$ & Hydroxychloroquine & $\begin{array}{l}\text { Lopinavir/ritonavir } \\
\text { Baricitinib } \\
\text { Sarilumab }\end{array}$ & $\begin{array}{l}\text { Clinical status } \\
\text { Mortality } \\
\text { Length of disease }\end{array}$ & $\begin{array}{l}\text { Nova Scotia } \\
\text { Health Authority }\end{array}$ \\
\hline ChiCTR2000030718 & Recruiting & Not reported & RCT & $\begin{array}{l}\text { Participants with } \\
\text { confirmed } \\
\text { COVID-19 } \\
\text { diagnosis } \\
(80)\end{array}$ & Hydroxychloroquine & No intervention & $\begin{array}{l}\text { Mortality } \\
\text { Severity of } \\
\text { respiratory } \\
\text { impairment } \\
\text { Length of disease } \\
\text { Laboratory } \\
\text { detection/viral load } \\
\text { Oxygen } \\
\text { supplementation } \\
\text { duration }\end{array}$ & $\begin{array}{l}\text { Hubei Clinical } \\
\text { Research Center } \\
\text { for Emergency } \\
\text { and Resuscitation }\end{array}$ \\
\hline ChiCTR2000029988 & Recruiting & Not reported & RCT & $\begin{array}{l}\text { Participants with } \\
\text { confirmed } \\
\text { COVID-19 } \\
\text { diagnosis and } \\
\text { severe disease } \\
\text { (80) }\end{array}$ & Chloroquine & No intervention & $\begin{array}{l}\text { All-cause mortality } \\
\text { Length of } \\
\text { hospitalization } \\
\text { Length of ICU stay } \\
\text { Length of } \\
\text { mechanical } \\
\text { ventilation }\end{array}$ & $\begin{array}{l}\text { Hubei Clinical } \\
\text { Research Center } \\
\text { for Emergency } \\
\text { and Resuscitation }\end{array}$ \\
\hline ChiCTR2000029939* & Recruiting & Not reported & RCT & $\begin{array}{l}\text { Participants with } \\
\text { confirmed } \\
\text { COVID-19 } \\
\text { diagnosis } \\
(100)\end{array}$ & Chloroquine & No intervention & $\begin{array}{l}\text { Specific mortality } \\
\text { Length of } \\
\text { hospitalization }\end{array}$ & $\begin{array}{l}\text { HwaMei Key } \\
\text { Research Fund } \\
\text { (2020HMZD18) }\end{array}$ \\
\hline ChicTR2000029935* & Recruiting & Not reported & $\begin{array}{l}\text { Single } \\
\text { arm } \\
\text { study }\end{array}$ & $\begin{array}{l}\text { Participants with } \\
\text { confirmed } \\
\text { COVID-19 } \\
\text { diagnosis } \\
(100)\end{array}$ & Chloroquine & NA & $\begin{array}{l}\text { Specific mortality } \\
\text { específica }\end{array}$ & $\begin{array}{l}\text { HwaMei Key } \\
\text { Research Fund } \\
\text { (2020HMZD18) }\end{array}$ \\
\hline
\end{tabular}


Chart 2. Characteristics and methodological aspects of the ongoing studies (conclusion)

\begin{tabular}{|c|c|c|c|c|c|c|c|c|}
\hline Study & Status & $\begin{array}{c}\text { Estimate } \\
\text { start/end date }\end{array}$ & Design & Participants (n) & Intervention & Comparators & $\begin{array}{c}\text { Main interest } \\
\text { outcomes }\end{array}$ & Funding \\
\hline $\begin{array}{l}\text { ChicTR2000029899/ } \\
\text { ChicTR2000029898 }\end{array}$ & Recruiting & Not reported & RCT & $\begin{array}{l}\text { Participants with } \\
\text { confirmed } \\
\text { COVID-19 } \\
\text { diagnosis } \\
(100)\end{array}$ & Hydroxychloroquine & Chloroquine & $\begin{array}{l}\text { Length of disease } \\
\text { All-cause mortality }\end{array}$ & $\begin{array}{l}\text { Peking University } \\
\text { Third Hospital }\end{array}$ \\
\hline ChicTR2000029868 & Recruiting & Not reported & RCT & $\begin{array}{l}\text { Participants with } \\
\text { confirmed } \\
\text { COVID-19 } \\
\text { diagnosis } \\
(200)\end{array}$ & Hydroxychloroquine & No intervention & $\begin{array}{l}\text { Laboratory } \\
\text { detection/viral load }\end{array}$ & $\begin{array}{l}\text { SPH SHANGHAI } \\
\text { ZHONGXI } \\
\text { PHARMACEUTICAL } \\
\text { CO., LTD }\end{array}$ \\
\hline ChiCTR2000029741 & Recruiting & Not reported & RCT & $\begin{array}{l}\text { Participants with } \\
\text { confirmed } \\
\text { COVID-19 } \\
\text { diagnosis } \\
(112)\end{array}$ & Hydroxychloroquine & Lopinavir / Ritonavir & $\begin{array}{l}\text { Length of } \\
\text { hospitalization } \\
\text { Proportion of } \\
\text { critical cases } \\
\text { All-cause mortality }\end{array}$ & $\begin{array}{l}\text { Sun Yat-Sen } \\
\text { University }\end{array}$ \\
\hline ChicTR2000029740 & Recruiting & Not reported & RCT & $\begin{array}{l}\text { Participants with } \\
\text { confirmed } \\
\text { COVID-19 } \\
\text { diagnosis } \\
(78)\end{array}$ & Hydroxychloroquine & No intervention & $\begin{array}{l}\text { Laboratory } \\
\text { detection/viral load }\end{array}$ & $\begin{array}{l}\text { The First Hospital } \\
\text { of Peking } \\
\text { University }\end{array}$ \\
\hline ChicTR2000029559 & Recruiting & Not reported & RCT & $\begin{array}{l}\text { Participants with } \\
\text { confirmed } \\
\text { cOVID-19 } \\
\text { diagnosis } \\
(200)\end{array}$ & Hydroxychloroquine & Placebo & $\begin{array}{l}\text { Laboratory } \\
\text { detection/viral load }\end{array}$ & $\begin{array}{l}\text { Renmin Hospital } \\
\text { of Wuhan } \\
\text { University }\end{array}$ \\
\hline ChiCTR2000029542 & Recruiting & Not reported & RCT & $\begin{array}{l}\text { Participants with } \\
\text { confirmed } \\
\text { covID-19 } \\
\text { diagnosis } \\
(20)\end{array}$ & Chloroquine & No intervention & $\begin{array}{l}\text { All-cause mortality } \\
\text { Length of ICU stay } \\
\text { Length of } \\
\text { hospitalization }\end{array}$ & $\begin{array}{l}\text { Sun Yat sen } \\
\text { Memorial Hospital } \\
\text { of Sun Yat sen } \\
\text { University }\end{array}$ \\
\hline NCT04321278 & $\begin{array}{l}\text { Not yet } \\
\text { recruiting }\end{array}$ & $\begin{array}{l}23 \text { March } \\
2020 / 30 \text { August } \\
2020\end{array}$ & RCT & $\begin{array}{l}\text { Participants with } \\
\text { likely or } \\
\text { confirmed } \\
\text { COVID-19 } \\
\text { diagnosis } \\
(440)\end{array}$ & Hydroxychloroquine & $\begin{array}{l}\text { Hydroxychloroquine } \\
+ \text { Azithromycin }\end{array}$ & $\begin{array}{l}\text { Clinical status } \\
\text { All-cause mortality } \\
\text { Length of } \\
\text { hospitalization } \\
\text { Number of days } \\
\text { without } \\
\text { mechanical } \\
\text { ventilation }\end{array}$ & $\begin{array}{l}\text { Hospital Israelita } \\
\text { Albert Einstein }\end{array}$ \\
\hline NCT04303299 & $\begin{array}{l}\text { Not yet } \\
\text { recruiting }\end{array}$ & $\begin{array}{l}15 \text { March } \\
2020 / 30 \\
\text { November } 2020\end{array}$ & RCT & $\begin{array}{l}\text { Participants with } \\
\text { confirmed } \\
\text { COVID-19 } \\
\text { diagnosis } \\
\text { (80) }\end{array}$ & $\begin{array}{l}\text { Oseltamivir + } \\
\text { Chloroquine }\end{array}$ & $\begin{array}{l}\text { Different schemes } \\
\text { of Oseltamivir, } \\
\text { Darunavir, Lopinavir } \\
\text { and Faviparivir }\end{array}$ & $\begin{array}{l}\text { Laboratory } \\
\text { detection/viral load } \\
\text { Mortality } \\
\text { Length of } \\
\text { mechanical } \\
\text { ventilation }\end{array}$ & Rajavithi Hospital \\
\hline NCT04322123 & $\begin{array}{l}\text { Not yet } \\
\text { recruiting }\end{array}$ & $\begin{array}{l}6 \text { April 2020/30 } \\
\text { August } 2020\end{array}$ & RCT & $\begin{array}{l}\text { Participants with } \\
\text { likely or } \\
\text { confirmed } \\
\text { COVID-19 } \\
\text { diagnosis } \\
(630)\end{array}$ & Hydroxychloroquine & $\begin{array}{l}\text { Hydroxychloroquine } \\
+ \text { Azithromycin }\end{array}$ & $\begin{array}{l}\text { Clinical status } \\
\text { All-cause mortality } \\
\text { Length of } \\
\text { hospitalization } \\
\text { Proportion of } \\
\text { patients with } \\
\text { orotracheal } \\
\text { intubation }\end{array}$ & $\begin{array}{l}\text { Hospital do } \\
\text { Coração }\end{array}$ \\
\hline NCT04324463 & $\begin{array}{l}\text { Not yet } \\
\text { recruiting }\end{array}$ & $\begin{array}{l}1 \text { April 2020/30 } \\
\text { September } \\
2020\end{array}$ & $\mathrm{RCT}$ & $\begin{array}{l}\text { Participants with } \\
\text { confirmed } \\
\text { COVID-19 } \\
\text { diagnosis } \\
(1500)\end{array}$ & $\begin{array}{l}\text { Chloroquine + } \\
\text { Azithromycin }\end{array}$ & No intervention & $\begin{array}{l}\text { Hospitalization or } \\
\text { death } \\
\text { Mechanical } \\
\text { ventilation or } \\
\text { death }\end{array}$ & $\begin{array}{l}\text { Population Health } \\
\text { Research Institute }\end{array}$ \\
\hline NCT04304053 & Recruiting & $\begin{array}{l}18 \text { March } \\
2020 / 15 \text { June } \\
2020\end{array}$ & RCT & $\begin{array}{l}\text { Participants with } \\
\text { confirmed } \\
\text { COVID-19 } \\
\text { diagnosis } \\
(3040)\end{array}$ & $\begin{array}{l}\text { Hydroxychloroquine } \\
\text { + Darunavir }\end{array}$ & No intervention & $\begin{array}{l}\text { Incidence of } \\
\text { COVID-19 in close } \\
\text { contacts }\end{array}$ & $\begin{array}{l}\text { Fundacio Lluita } \\
\text { Contra la SIDA }\end{array}$ \\
\hline NCT04322396 & $\begin{array}{l}\text { Not yet } \\
\text { recruiting }\end{array}$ & $\begin{array}{l}1 \text { April 2020/31 } \\
\text { October } 2020\end{array}$ & $\mathrm{RCT}$ & $\begin{array}{l}\text { Participants with } \\
\text { confirmed } \\
\text { COVID-19 } \\
\text { diagnosis } \\
(226)\end{array}$ & $\begin{array}{l}\text { Hydroxychloroquine } \\
+ \text { Azithromycin }\end{array}$ & Placebo & $\begin{array}{l}\text { Clinical status } \\
\text { Mortality } \\
\text { Length of } \\
\text { hospitalization }\end{array}$ & $\begin{array}{l}\text { Chronic } \\
\text { Obstructive } \\
\text { Pulmonary } \\
\text { Disease Trial } \\
\text { Network, } \\
\text { Denmark }\end{array}$ \\
\hline NCT04323527 & Recruiting & $\begin{array}{l}23 \text { March } \\
2020 / 31 \text { August } \\
2020\end{array}$ & RCT & $\begin{array}{l}\text { Participants with } \\
\text { likely or } \\
\text { confirmed } \\
\text { COVID-19 } \\
\text { diagnosis } \\
(440)\end{array}$ & $\begin{array}{l}\text { Chloroquine (high } \\
\text { dosage) }\end{array}$ & $\begin{array}{l}\text { Chloroquine (low } \\
\text { dosage) }\end{array}$ & $\begin{array}{l}\text { Mortality } \\
\text { Length of } \\
\text { hospitalization } \\
\text { Length of } \\
\text { mechanical } \\
\text { ventilation }\end{array}$ & $\begin{array}{l}\text { Fundação de } \\
\text { Medicina Tropical } \\
\text { Dr. Heitor Vieira } \\
\text { Dourado }\end{array}$ \\
\hline
\end{tabular}



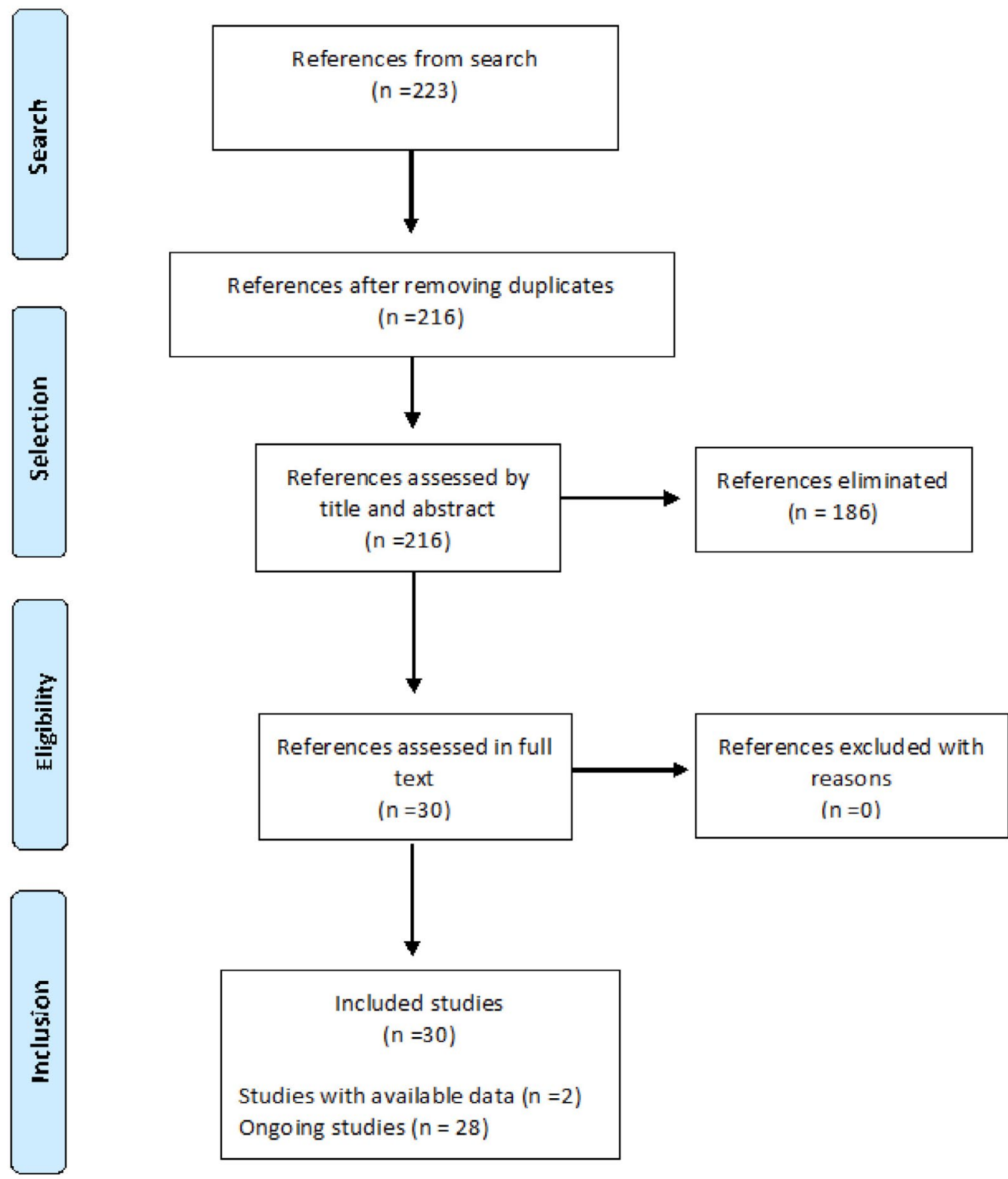

Main findings and methodological characteristics of the two clinical studies with available results are depicted in Chart 3. Details of the 28 ongoing studies are presented in the Chart 2.

The two included studies evaluated the same primary outcome (viral detection), in a similar follow-up ( 6 and 7 days $)^{10,11}$. However, we did not consider appropriate to perform a quantitative synthesis of its results (meta-analysis) due to the following aspects related to the clinical and methodological heterogeneity between the studies:

- Different study design: randomized clinical trial10 and non-randomized clinical trial ${ }^{11}$.

- Different methods of data analysis: intention-totreat ${ }^{10}$ and per protocol ${ }^{11}$.
- Different hydroxychloroquine dosage and treatment duration: $400 \mathrm{mg} /$ day for five days ${ }^{10}$ and $600 \mathrm{mg} /$ day for 10 days ${ }^{11}$.

- Difference in the mean age of participants in the control group: $50.5 \pm 3.8^{10}$ and $37.3 \pm 24.0^{11}$. The difference in the standard deviation between the groups also shows that the age dispersion was lower in Chen 2020, increasing heterogeneity between the samples from the two studies.

- Differences in the frequency of the primary outcome in the control groups: $93.3 \%{ }^{10}$ and $12.5 \%{ }^{11}$. This difference may indicate that the population from both studies were not similar and/or the cointerventions used were different, impacting on the effects observed from the intervention.

- Differences in co-interventions allowed during the study conduction. 
Chart 3. Main findings and methodological characteristics of the included studies (to be continued)

\begin{tabular}{|c|c|c|}
\hline Study & Chen $2020^{10}$ & Gautret $2020^{11}$ \\
\hline Design & $\begin{array}{l}\text { Open-label randomized clinical trial } \\
\text { (NCT04261517) }\end{array}$ & $\begin{array}{l}\text { Open label non- randomized clinical trial } \\
\text { (EU Clinical Trials Register 2020-000890-25) }\end{array}$ \\
\hline $\begin{array}{l}\text { Population } \\
\text { /condition of } \\
\text { interest }\end{array}$ & $\begin{array}{l}\text { - Hospitalized patients with documented } \\
\text { diagnosis of COVID-19 infection } \\
\text { - > } 18 \text { years old }\end{array}$ & $\begin{array}{l}\text { - } \begin{array}{l}\text { Hospitalized patients with } \\
\text { documented diagnosis of COVID-19 } \\
\text { infection }\end{array} \\
\text { - }>12 \text { years old }\end{array}$ \\
\hline Interventions & $\begin{array}{l}\text { - Hydroxychloroquine } 400 \mathrm{mg} / 1 \mathrm{x} \text { day for } \\
5 \text { days }(n=15) \\
\text { - Standard treatment }(n=15)\end{array}$ & $\begin{array}{l}\text { - Hydroxychloroquine } 200 \mathrm{mg} \text { - } 3 \\
\text { times / day for } 10 \text { days }(n=20) \\
\text { times / day for } 10 \text { days associated with } \\
\text { azithromycin }(500 \mathrm{mg} 1 \times \text { / day }+250 \mathrm{mg} \text { / } \\
\text { day for } 4 \text { days) }(n=6) \\
\text { Standard treatment }(n=16)\end{array}$ \\
\hline Funding & Shanghai Public Health Clinical Center & French Government \\
\hline $\begin{array}{l}\text { Negative viral } \\
\text { load in } \\
\text { oropharyngeal } \\
\text { swab by PCR }\end{array}$ & $\begin{array}{l}\text { - After } 7 \text { days of treatment: } 86.7 \% \\
(13 / 15) \text { in the hydroxychloroquine } \\
\text { group had negative viral detection } \\
\text { versus } 93.3 \%(14 / 15) \text { in the control } \\
\text { group ( } p>0.05) \\
\text { - } \quad \text { After } 14 \text { days, all } 30 \text { patients had a } \\
\text { negative test. }\end{array}$ & $\begin{array}{l}\text { - After } 6 \text { days of treatment: } 70 \% \text { of the } \\
\text { hydroxychloroquine group had no } \\
\text { viral detection versus 12.5\% in the } \\
\text { control group ( } p=0.001 \text { ) } \\
\text { - Post-hoc analysis: } 100 \% \text { of the } \\
\text { hydroxychloroquine + azithromycin } \\
\text { group }(n=6) \text { had no viral detection } \\
\text { versus } 57.1 \% \text { in the } \\
\text { hydroxychloroquine group versus } \\
12.5 \% \text { in the standard treatment } \\
\text { group. }\end{array}$ \\
\hline Adverse events & $\begin{array}{l}\text { - Hydroxychloroquine group: four } \\
\text { events: } \\
\circ \text { Diarrhea }(n=2) \\
\circ \text { Worsening of the clinical } \\
\text { condition with discontinuation of } \\
\text { treatment }(n=1) \\
\circ \text { Transient increase in aspartate } \\
\text { aminotransferase }(n=1) \\
\text { Standard treatment group: } 3 \text { events } \\
\circ \text { Increase in serum creatinine }(n \\
=1) \\
\circ \text { Anemia }(n=1) \\
\circ \text { Transient increase in aspartate } \\
\text { aminotransferase }(n=1) .\end{array}$ & Not assessed \\
\hline $\begin{array}{l}\text { Time to negative } \\
\text { viral load (PCR) }\end{array}$ & $\begin{array}{l}\text { - Hydroxychloroquine group: median } 4 \\
\text { days (1st quartile = 1; 3rd quartile = 9) } \\
\text { - Standard treatment group: Median } 2 \\
\text { days (1st quartile = 1; 3rd quartile = } \\
\text { 4). }\end{array}$ & Not assessed \\
\hline
\end{tabular}


Chart 3. Main findings and methodological characteristics of the included studies (conclusion)

\begin{tabular}{|c|c|c|}
\hline Study & Chen $2020^{10}$ & Gautret $2020^{11}$ \\
\hline $\begin{array}{l}\text { Radiological } \\
\text { progression }\end{array}$ & $\begin{array}{l}\text { - Hydroxychloroquine group: } 33 \% \\
\text { (5/15) presented radiological } \\
\text { improvement after } 3 \text { days of follow- } \\
\text { up and } 100 \% \text { after } 14 \text { days. } \\
\text { - Standard treatment: } 46.7 \% \text { presented } \\
\text { radiological improvement ( } 7 / 15) \text { after } \\
3 \text { days of follow-up and } 100 \% \text { after } 14 \\
\text { days. }\end{array}$ & Not assessed \\
\hline Mortality & $\begin{array}{l}\text { There were no deaths in either intervention } \\
\text { groups at } 14 \text { days of follow-up }\end{array}$ & Not reported \\
\hline Risk of bias & $\begin{array}{l}\text { High risk of performance bias and unclear risk } \\
\text { of selection and detection bias }\end{array}$ & Overall risk of bias: serious \\
\hline
\end{tabular}

PCR: polymerase chain reaction

\section{Risk of bias assessment of the included studies}

The risk of bias assessment of the included studies and all justifications for each judgment is presented in Charts 4 and 5.

Chart 4. Risk of bias of the included randomized clinical trial ${ }^{10}$, using the Cochrane Risk of Bias Table ${ }^{4}$

\begin{tabular}{|c|c|c|}
\hline Domain & Judgment & Commentaries and justifications \\
\hline $\begin{array}{l}\text { Random sequence } \\
\text { generation }\end{array}$ & Unclear & Not reported \\
\hline Allocation concealment & Unclear & Not reported \\
\hline $\begin{array}{l}\text { Blinding of participants and } \\
\text { personnel }\end{array}$ & High risk & Open label study \\
\hline $\begin{array}{l}\text { Blinding of outcome } \\
\text { assessors }\end{array}$ & Unclear & It is not clear if the outcome assessor was blinded. \\
\hline Incomplete outcome data & Low & $\begin{array}{l}\text { Only one participant in the intervention arm had to } \\
\text { stop taking hydroxychloroquine due to adverse events. } \\
\text { The authors performed an intention- to-treat analysis. }\end{array}$ \\
\hline Selective outcome reporting & Low & $\begin{array}{l}\text { The clinicaltrials.gov registry (NCTO4261517) was } \\
\text { published on 7th February, } 2020 \text { and the enrollment } \\
\text { period was from 6th February } 2020 \text { to 25th February } \\
\text { 2020. Despite this delay in one day in the registry, } \\
\text { considering the number of participants and the } \\
\text { extension of the enrollment period it was considered } \\
\text { that this registry was prospective. The primary } \\
\text { outcomes were pre-planned and reported in the } \\
\text { manuscript. }\end{array}$ \\
\hline Other bias & Low & We did not identify any other apparent source of bias. \\
\hline
\end{tabular}


Chart 5. Risk of bias of the non-randomized clinical trial ${ }^{11}$, using the ROBINS-II ${ }^{7}$

\begin{tabular}{|c|c|c|}
\hline Domain & Judgment & Commentaries and justifications \\
\hline Bias due to confounding & Serious & $\begin{array}{l}\text { The baseline mean age of the participants in the hydroxychloroquine } \\
\text { group was } 51.2 \text { years (standard deviation 18.7) and } 37.3 \text { (standard } \\
\text { deviation } 24.0 \text { ) in the control group. The authors reported a p value = } \\
0.06 \text {. However, this is an important imbalance in this prognosis factor. } \\
\text { The fact that the mean age was higher in the intervention group may } \\
\text { indicate that it was preferred to include patients with higher risk factors } \\
\text { in the intervention group. }\end{array}$ \\
\hline $\begin{array}{l}\text { Bias in selection of } \\
\text { participants into the } \\
\text { study }\end{array}$ & Serious & $\begin{array}{l}\text { The intervention group was recruited in a single centre ("The } \\
\text { Méditerranée Infection University Hospital Institute in Marseille") and the } \\
\text { control group was recruited in other centres ("Controls without } \\
\text { hydroxychloroquine treatment were recruited in Marseille, Nice, Avignon } \\
\text { and Briançon centers, all located in South France"). It is also stated that } \\
\text { patients in the Marseille group that refused consent or were not eligible } \\
\text { to receive hydroxychloroquine were used as controls. This characteristic } \\
\text { increases the risk of bias expressively, because co-interventions and the } \\
\text { conditions of each centre can be very distincts, generating an imbalance } \\
\text { in the baseline and during the evolution of the study. }\end{array}$ \\
\hline $\begin{array}{l}\text { Bias in classification of } \\
\text { interventions }\end{array}$ & Low & $\begin{array}{l}\text { This was a prospective study. The risk of bias related to classification of } \\
\text { interventions is low. }\end{array}$ \\
\hline $\begin{array}{l}\text { Bias due to deviations } \\
\text { from intended } \\
\text { interventions }\end{array}$ & Serious & $\begin{array}{l}\text { This was an open-label study. Six patients in the hydroxychloroquine } \\
(23 \%) \text { also received azithromycin. Besides that, co-interventions were } \\
\text { not controlled and probably not homogenous between the arms of the } \\
\text { trial. }\end{array}$ \\
\hline Bias due to missing data & Serious & $\begin{array}{l}\text { Six patients in the hydroxychloroquine ( } 23 \%) \text { group were not analyzed } \\
\text { for the reported outcome. Despite being reported as "losses to follow- } \\
\text { up", the authors performed a per protocol analysis. }\end{array}$ \\
\hline $\begin{array}{l}\text { Bias in measurement of } \\
\text { outcomes }\end{array}$ & Moderate & $\begin{array}{l}\text { It was not clear if the outcome assessor was blinded. Despite the } \\
\text { outcome is laboratorial, the procedures to collect the samples may be } \\
\text { different and influenced by the enrolment awareness. }\end{array}$ \\
\hline $\begin{array}{l}\text { Bias in selection of the } \\
\text { reported result }\end{array}$ & Serious & $\begin{array}{l}\text { Planned outcomes were not reported and the time point reported in } \\
\text { the study ( } 6 \text { days) was not planned in the protocol [EU Clinical Trials } \\
\text { Register 2020-000890-25]. }\end{array}$ \\
\hline Overall bias & Serious & The study was considered to be at serious risk of bias in five domains. \\
\hline
\end{tabular}

\section{Assessment of the evidence certainty}

We used the GRADE approach to assess the evidence provided by the randomized clinical trial included in this review (Table 1). For all considered outcomes, the evidence was graded as very low certainty (downgraded two levels due to imprecision and two levels due to risk of bias). This means that we are uncertain regarding any effect that hydroxychloroquine may have in COVID-19 patients, and future studies are likely to change any efficacy and safety estimates reported by the study. 

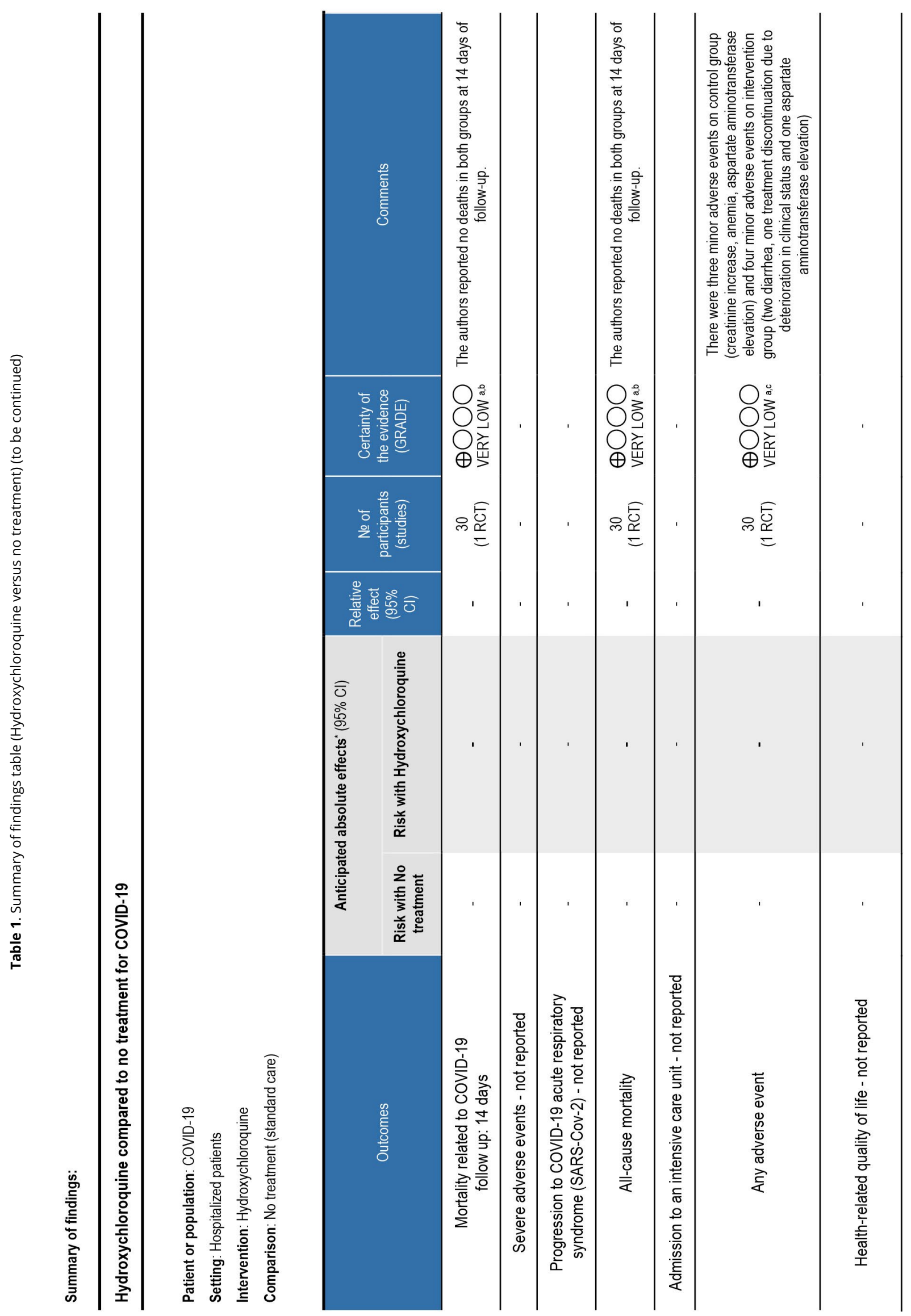

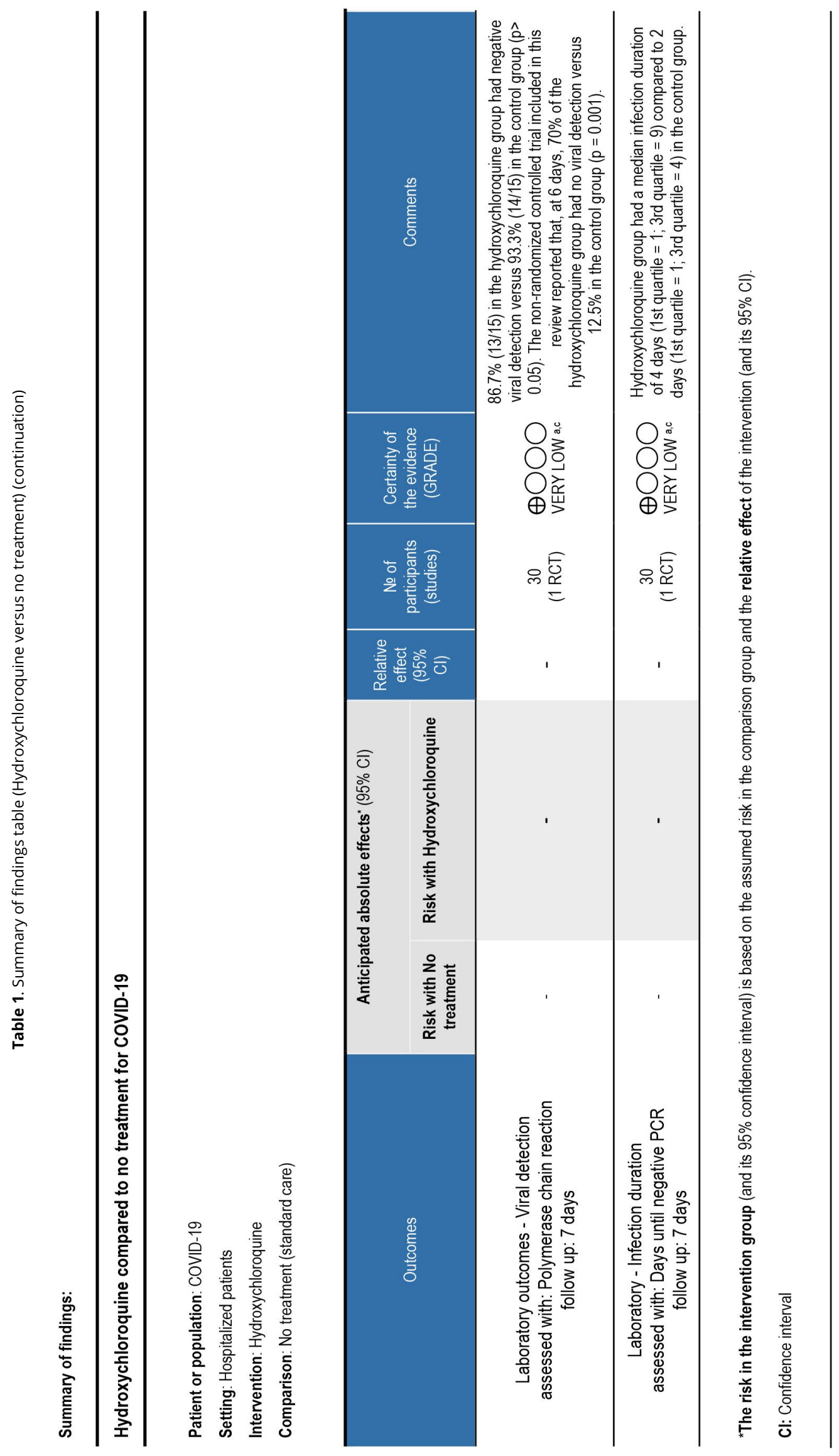


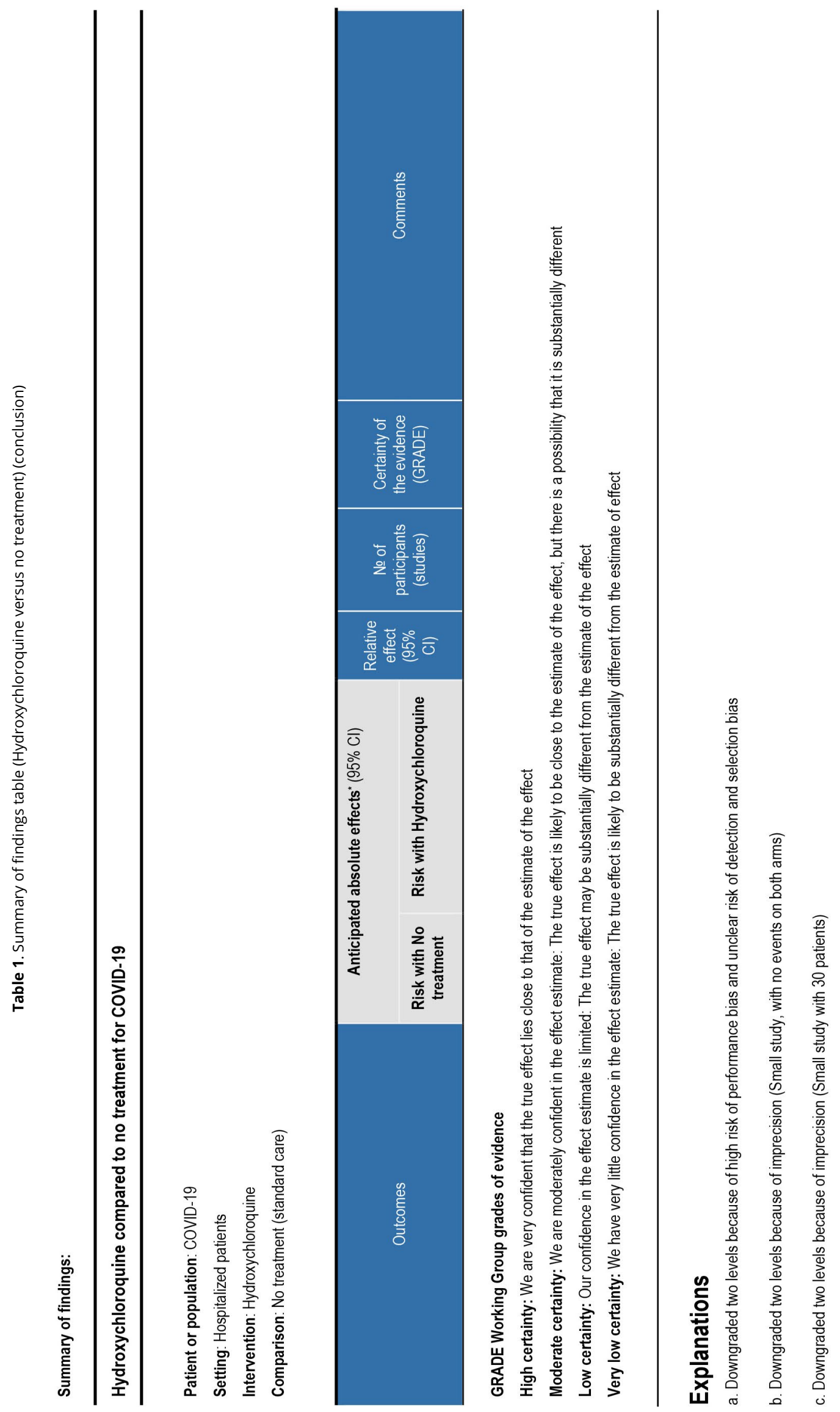




\section{Discussion}

Although experimental in vitro studies suggest potential antiviral action of hydroxychloroquine against COVID-1912,13, and health authorities are recommending this drug for patients with COVID-19 infection, this rapid systematic review identified mere two studies with available data addressing this question: one open-label non-randomized trial with 42 participants 11 and one open-label randomized trial with 30 participants ${ }^{10}$. These studies present high risk of bias and their results were inconsistent about the only common outcome between them (viral load detection).

There are currently 58 cataloged biases that can contribute to the results of clinical studies to distance themselves from the truth ${ }^{14,15}$. In order to have reliable and applicable results, it is expected that rigorous methods will be adopted by studies to prevent the occurrence of these biases during the planning, conducting and reporting phases. However, this methodological rigor, already known since the conduct of the first clinical trial, was not adopted by the two studies presented.

The presence of a similar comparator group (which probably did not occur in the studies) is essential to estimate the real effects of hydroxychloroquine and to assess whether these effects are different from those observed using the best available option, placebo or natural course of the disease. This similarity also contributes to assure that the estimated effect may be due exclusively to the intervention, eliminating the effect of confounding factors present at different levels in the compared groups, such as disease severity, age or comorbidities. An appropriate randomization method would avoid this confusion. Although the Chen 2020 study is described as randomized, details of randomization and methods to maintain allocation concealment were not presented by the authors.

The absence of adequate methods for allocation concealment could overestimate the effect of the intervention by $37 \%$ to $41 \% \frac{16}{16}$. That is, depending on the size of the effect, an estimate that means a benefit may actually be wrong.
The lack of masking of participants, personnel and outcomes assessors can lead to deviations in the process of conducting the study (such as impacting on the adherence to treatment and notification of adverse events) and in the process of evaluating the outcomes.

The existence of favorable recommendations from some parties involved in the decision-making process regarding the use of hydroxychloroquine underscores the importance of the results of ongoing trials so that the effects of hydroxychloroquine for patients with COVID-19 are known. This is a point that needs to be addressed in a context where there is an urgent need for answers. As this review identified 28 approved clinical trial records in progress on the two largest clinical trial platforms (Clinicaltrials.gov and ICTRPWHO), more data will be available soon.

Finally, the justification for the use of drugs for cases of COVID-19, as well as for any other disease, must be based on the existence of clinical benefits (reduced mortality, and respiratory complications for example) observed through reliable clinical studies, preferably randomized, double-blind clinical trials.

The use of a drug should not be justified solely by its potential mechanisms of action observed in experimental / preclinical studies. Recent examples, such as the use of albumin in large burns, have already shown that this is not an acceptable strategy when the objective is to offer a treatment with a better probability of benefits than harms.

Ignoring these precepts certainly increases uncertainty in decision-making - which means the exact opposite of what clinical research has sought to follow, more rigorously, over the past 25 years. Thus, in view of the alarming current scenario, it is essential that decisions are informed by the best available evidence, so that today's actions are more likely to bring benefits than harms to the population.

As implication for practice, this review did not find sufficient evidence to support the use of hydroxychloroquine or chloroquine as a routine for treating all patients with COVID-19, neither for preventive purposes. The exceptional prescription should be restricted for those patients with severe cases of COVID-19 infection, who are not at increased risk of adverse events associated with the use of hydroxychloroquine, and within a context of scientific investigation. 


\section{Conclusion}

This rapid systematic review identified two clinical studies (with available data), with limited methodological quality, that evaluated the effects of hydroxychloroquine for COVID-19. Based on the findings of these studies, the efficacy and safety of hydroxychloroquine and chloroquine in patients with COVID-19 is still uncertain and its routine use for this situation should not be recommended until the results of ongoing studies could provide a proper assessment of their effects.

\section{Author contributions}

Both authors designed and performed the review, wrote the report and approved the final version.

\section{Competing interests}

No financial, legal or political competing interests with third parties (government, commercial, private foundation, etc.) were disclosed for any aspect of the submitted work (including but not limited to grants, data monitoring board, study design, manuscript preparation, statistical analysis, etc.).

\section{References}

1. China law translate. Novel Coronavirus Pneumonia Diagnosis and Treatment Plan (Provisional 7th Edition). [Internet]. 2020. [acesso em 2020 mar. 26]. Disponível em: https://www. chinalawtranslate.com/coronavirus-treatment-plan-7/

2. Yao X, Ye F, Zhang M, Cui C, Huang B, Niu P et al. In Vitro Antiviral Activity and Projection of Optimized Dosing Design of Hydroxychloroquine for the Treatment of Severe Acute Respiratory Syndrome Coronavirus 2 (SARS-CoV-2). Clinical Infectious Diseases: An Official Publication of the Infectious Diseases Society of America. 2020. doi: 10.1093/cid/ciaa237

3. Sung-sun K. Physicians work out treatment guidelines for coronavirus". m.koreabiomed.com (in Korean). [Internet]. 2020. [acesso em 2020 mar. 26]. Disponível em: http://m.koreabiomed. com/news/articleView.html?idxno=7428.

4. Higgins JPT, Thomas J, Chandler J, Cumpston M, Li T, Page MJ et al (editors). Cochrane Handbook for Systematic Reviews of Interventions version 6.0 (updated July 2019). [Internet]. 2019. [acesso em 2020 mar. 26]. Disponível em: www.training.cochrane. org/handbook
5. Moher D, Liberati A, Tetzlaff J, Altman DG, The PRISMA Group (2009). Preferred Reporting Items for Systematic Reviews and Meta-Analyses: The PRISMA Statement. PLoS Med. 2009;21;6(7):e1000097. doi: 10.1371/journal.pmed.1000097

6. Ouzzani M, Hammady H, Fedorowicz Z, Elmagarmid A. Rayyan - a web and mobile app for systematic reviews. Syst. Rev. 2016;5(1):210. doi: 10.1186/s13643-016-0384-4

7. Stern JAC, Herńan MA, Reeves BC, Savoić J, Berkman ND, Viswanathan $M$ et al. ROBINS-I: a tool for assessing risk of bias in non-randomised studies of intervention. BMJ. 2016;355:i4919. doi: $\underline{10.1136 / \mathrm{bmj} . i 4919}$

8. Wells GA, Shea B, O'Connell D, Peterson J, Welch V, Losos $M$ et al. The Newcastle-Ottawa Scale (NOS) for assessing the quality if nonrandomized studies in meta-analyses. [Internet]. 2012. [acesso em 2020 mar. 26]. Disponível em: http://ohri.ca/ programs/clinical_epidemiology/oxford.htm

9. Guyatt GH, Oxman AD, Vist GE, Kunz R, Falck-Ytter Y, AlonsoCoello $P$ et al. GRADE: an emerging consensus on rating quality of evidence and strength of recommendations. BMJ. 2008;336(7650):924-6. doi: 10.1136/bmj.39489.470347

10. Chen J, Liu D, Li Liu, LIU Ping,XU Qingnian,XIA Lu et al. A pilot study of hydroxychloroquine in treatment of patients with common coronavirus disease-19 (COVID-19). J Zhejiang Univ (Med Sci). 2020;49(1). doi: 10.3785/j.issn.1008-9292.2020.03.03

11. Gautret P, Lagiera JC, Parola P, Hoang VT, Meddeba L, Mailhea $M$ et al. Hydroxychloroquine and azithromycin as a treatment of COVID-19: results of an open-label non-randomized clinical trial. International Journal of Antimicrobial Agents. 2020. doi: 10.1016/j. ijantimicag.2020.105949

12. Gao J, Zhenxue T, Xu Y. Breakthrough: Chloroquine phosphate has shown apparent efficacy in treatment of COVID-19 associated pneumonia in clinical studies. BioScience Trends. 2020;14(1):7273. doi: $\underline{10.5582 / \mathrm{bst} .2020 .01047}$

13. Liu J, Cao R, Xu M, Wang X, Zhang H, Hu Het al. Hydroxychloroquine, a less toxic derivative of chloroquine, is effective in inhibiting SARS-CoV-2 infection in vitro. Cell Discov. 2020;(6):16. doi: 10.1038/s41421-020-0156-0

14. Catalogue of Bias Collaboration. Catalogue Of Bias. [Internet]. 2020. [acesso em 2020 mar. 26]. Disponível em: https:// catalogofbias.org/biases/attrition-bias/

15. Pacheco RL, Latorraca COC, Martimbianco ALC, Fontes LES, Vieira R, Miranda E et al. Translation of Oxford's CEBM catalogue of bias into Portuguese: contributing to the dissemination of conscientious thinking on health research. BMJ. 2020. doi: $\underline{10.1136 / \mathrm{bmjebm}-2019-111329}$

16. Schulz KF, Chalmers I, Hayes RJ, Altman DG. Empirical evidence of bias. Dimensions of methodological quality associated with estimates of treatment effects in controlled trials. JAMA. 1995;273(5):408-12. doi: 10.1001/jama.273.5.408 